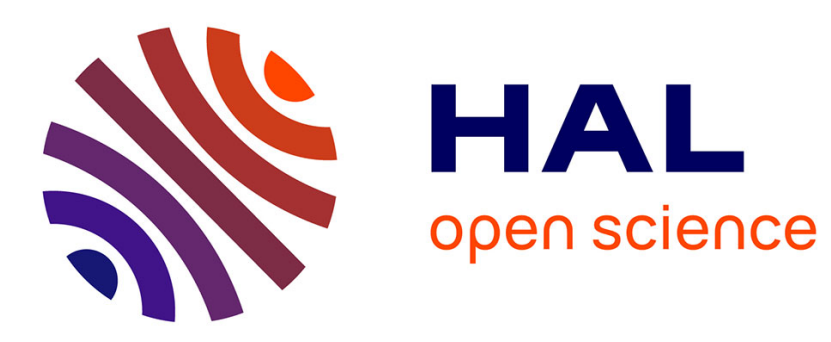

\title{
Implicit FEM and Fluid Coupling on GPU for Interactive Multiphysics Simulation
}

Jérémie Allard, Hadrien Courtecuisse, François Faure

\section{To cite this version:}

Jérémie Allard, Hadrien Courtecuisse, François Faure. Implicit FEM and Fluid Coupling on GPU for Interactive Multiphysics Simulation. SIGGRAPH Talks, Aug 2011, Vancouver, Canada. pp.Article No. $52,10.1145 / 2037826.2037895$. inria-00589203v2

\section{HAL Id: inria-00589203 https://hal.inria.fr/inria-00589203v2}

Submitted on 5 Apr 2012

HAL is a multi-disciplinary open access archive for the deposit and dissemination of scientific research documents, whether they are published or not. The documents may come from teaching and research institutions in France or abroad, or from public or private research centers.
L'archive ouverte pluridisciplinaire $\mathbf{H A L}$, est destinée au dépôt et à la diffusion de documents scientifiques de niveau recherche, publiés ou non, émanant des établissements d'enseignement et de recherche français ou étrangers, des laboratoires publics ou privés. 


\section{Implicit FEM and Fluid Coupling on GPU for Interactive Multiphysics Simulation}

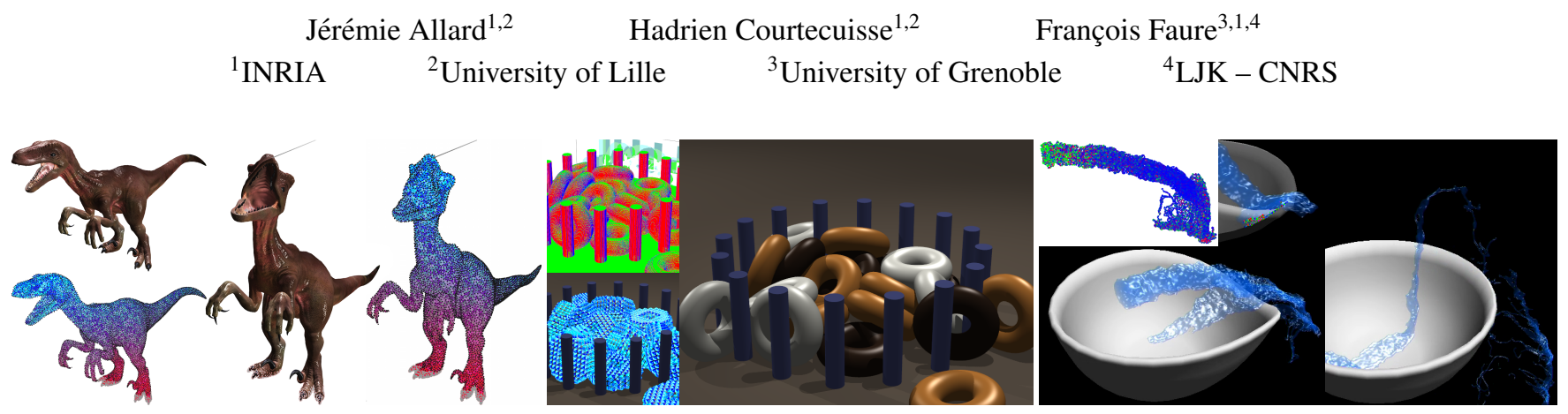

Figure 1: We combine GPU methods for detailed deformable objects (left), image-based collisions (middle), and SPH fluids to simulate a two-way fluid-deformable coupling (right) at interactive rates.

\begin{abstract}
We present a method to implement on the GPU an implicit FEM solver which is fast and stable enough to handle interactions and collisions. We combine this method with GPU-based fluids [Zhang et al. 2008] and collision detection [Allard et al. 2010] to achieve interactive multiphysics simulations entirely running on the GPU.
\end{abstract}

\section{Introduction}

The Finite Element Method (FEM) is widely used to simulate deformable materials in mechanical simulations. Recently, co-rotational linear FEM was successfully applied in interactive games [Mendoza and Garcia 2010] and medical simulations, however CPU methods are limited to coarse meshes due to performance constraints. GPU-based FEM methods have been proposed [Comas et al. 2008], but they apply explicit time integration and thus require prohibitively small time-steps. We overcome these limitations by implementing an implicit time integration scheme on the GPU [Allard et al. 2011]. GPU collision handling methods for rigid [Tonge et al. 2010] and deformable bodies [Allard et al. 2010] are available, yet an interactive simulation of two-way fluid-deformable coupling has remained an open problem.

\section{Methods}

Implicit FEM Solver on GPU To implement implicit FEM, we rely on an iterative Conjugate Gradient (CG) solver. However, in contrast to existing GPU-based sparse solvers [Krüger and Westermann 2005; Buatois et al. 2009], we do not explicitly build the system matrix, but instead parallelize the required matrix-vector products directly on the original mesh. This considerably reduces the number of operations required, and more importantly the consumed bandwidth, enabling the method to be fast enough for interactive simulations of soft bodies. The parallelization, detailed in [Allard et al. 2011], relies on first computing the contribution of mesh elements using one thread per tetrahedron, followed by a parallel gather to accumulate contributions at vertices. Further optimizations include mesh ordering, compact data structures, memory layout, and changing sequences of operations to reduce synchronization points.

Fluid Coupling using Image-based Collisions An imagebased collision method [Allard et al. 2010] has been proposed to handle complex deformable objects. It computes intersection volume gradients which are discretized on pixels and accumulated on vertices. To handle solid-fluid coupling, we extend this approach to compute additional pixels directly from the SPH fluid density field using ray-tracing. When intersections are detected, contributions are accumulated to the fluid particles based on their relative contribution as given by the SPH kernels evaluated at the pixel. As ray-tracing can be expensive, an important optimization is to test rays only when an existing pixel is within the fluid, which requires only a simple evaluation of the SPH density field.

\section{Results}

Our CUDA-based FEM solver is able to simulate a deformable object with 45k tetrahedral elements (Fig. 1 left) at 212 FPS on a Nvidia GeForce GTX 480, 18× faster than our most optimized sequential implementation on an Intel Core i7 $9753.33 \mathrm{GHz}$ CPU. All timings exclude rendering as the cost of this step can vary greatly depending on the desired visual quality and complexity. The fluid simulation (Fig. 1 right) demonstrates two-way coupling between the fluid and a soft cup, achieving 25 FPS using 2k FEM elements and 32k SPH particles on a GeForce GTX 280 GPU.

\section{References}

Allard, J., Faure, F., Courtecuisse, H., Falipou, F., DURIEZ, C., AND KRY, P. G. 2010. Volume contact constraints at arbitrary resolution. ACM Trans. Graph. 29, 4.

Allard, J., Courtecuisse, H., And Faure, F. 2011. Implicit FEM solver on GPU for interactive deformation simulation. In GPU Computing Gems Jade Edition. Elsevier, ch. 21. to appear.

Buatois, L., Caumon, G., And Lévy, B. 2009. Concurrent number cruncher - a GPU implementation of a general sparse linear solver. Int J Parallel Emerg. Distrib. Syst. 24, 3, 205-223.

Comas, O., Taylor, Z., Allard, J., Ourselin, S., Cotin, S., AND PASSENGER, J. 2008. Efficient nonlinear FEM for soft tissue modelling and its GPU implementation. In ISBMS, 28-39.

KrÜGer, J., AND Westermann, R. 2005. A GPU framework for solving systems of linear equations. In GPU Gems 2. Addison-Wesley, ch. 44, 703-718.

Mendoza, C., And Garcia, M. 2010. Soft bodies using finite elements. In Game Physics Pearls. A.K. Peters, ch. 10, 217-250.

Tonge, R., Wyatt, B., And Nicholson, N. 2010. PhysX GPU rigid bodies in Batman: Arkham Asylum. In Game Programming Gems 8. Cengage, ch. 7, 590-601.

Zhang, Y., Solenthaler, B., And Pajarola, R. 2008. Adaptive sampling and rendering of fluids on the GPU. In Proc. of Symp. on Point-Based Graph., 137-146. 